\title{
The Experiential Teaching of Berlin- Theoretical Reflections and Best Practices from a Study Abroad Site
}

\section{Thomas Wagenknecht}

\section{Humboldt University Berlin}

\section{Introduction}

Experiential learning plays a key role in enhancing intercultural sensitivity and competence within the context of international education. Ultimately, it is at the center of what leads the study abroad sojourn to become a positive and powerful learning process (Savicki, 2008). The academic teaching in study abroad programs frequently draws from the wide and diverse pool of material in and about the city in order to enable intercultural and experiential learning. Yet, creating first hand experiences of urban cultures that focus on reflection and leave profound impressions on students while providing the framing for future cycles of experiential learning is a complex and difficult dynamic to navigate (Kolb, 1984). The way urban manifestations of a different culture are directly experienced varies depending on the circumstances of a concrete cross-cultural encounter and the student's subjective interpretation of it.

To suggest a tentative framework within which the elusive nature of interpreting an urban culture can be channeled, facilitated, and made fertile for effective teaching, in this article I will turn to my on-going study conducted in Berlin, Germany. The specific study abroad program under investigation is designed around the study of the European metropolis. The city is approached from various academic angles-architecture, urban studies, visual arts, economics, gender, pop culture, European identity studies, and literary studies. For comparative purposes the program includes two extended field trips to Paris and St. Petersburg.

So far, both the analysis of interviews and the review of literature used in the study have produced conceptual components that inform a framework for facilitating the experiential teaching of urban cultures in intercultural education. However, it is still too early in the research process to yield a substantive theory for the experiential education of urban cultures. Hence, I will 
focus this article on the role and considerations of teachers who approach urban cultures by creating episodes of experiential learning.

First, I will elaborate on experiential learning theory and the philosophy of experiential education as two concepts that highlight complex teacherstudent-environment reciprocities. Second, the discussion of an interview, read against other interviews and the theory of experiential learning, will demonstrate the challenges of designing and conducting experiential learning situations. The ensuing discussion of best practices in mediating urban cultures will validate the city's capacity for experiential learning and reveal solutions for the intercultural hurdles involved in teaching "experientially." Ultimately, I will argue that more effort is required to close the gap between the theoretical and practical considerations of the experiential teaching of urban cultures in order to safeguard positive learning outcomes and increase the efficiency of teaching and learning in study abroad courses.

\section{From Experiential Learning to an Experiential Education in the City}

In his theory of experiential learning, Kolb defines learning as "the process whereby knowledge is created through the transformation of experience" (Kolb 1984, p.41). The model of the experiential learning cycle demonstrates a way in which one can grasp experience and, from it, structure a learning process. The model's appeal lies in its applicability to various circumstances ranging from Kolb's own classification of different learning styles, wherein the four nodes of the model - concrete experience, reflective observation, abstract conceptualization and active experimentation - are deconstructed, to Savicki's adaptation, in which he suggests interventions international educators can use to intervene in the cycle in an attempt to foster intercultural competence (Kolb, 2000; Savicki, 2008). Another appeal and, at the same time, essential connection to intercultural learning can be found in the learner's emotional investment in dealing with experiences (Cushner, 2004). With its focus on the learner's cognitive and emotional processes in actively engaging with present experiences and reflecting on them, the experiential learning cycle model can be complemented with Itin's transactional model of experiential education (Itin, 1999). To the changes in the learner based on direct experience, this model adds the transactions among teachers, learners, and the learning environment in which experiences are initi- 
ated and facilitated. Its underpinning philosophy of experiential education, which also provides a way of thinking about the study abroad experience per se, is based on

... carefully chosen experiences supported by reflection, critical analysis, and synthesis, [which] are structured to require the learner to take initiative, make decisions, and be accountable for the results through actively posing questions, investigating, experimenting, being curious, solving problems, assuming responsibility, being creative, constructing meaning and integrating previously developed knowledge. Learners are engaged intellectually, emotionally, socially, politically, spiritually, and physically in an uncertain environment where the learner may experience success, failure, adventure, and risk taking. (Itin, p.93)

This definition raises a central question; how can teachers deploy experiences to mediate between the city, which is the learning object as well as the environment, and students from a different cultural background? In order to approach this question, it is worthwhile to contemplate the dispositions the actors involved in the process of teaching/learning about the city bring to the table and how the complexity of the relationship among teacher, student, and urban manifestations is augmented by the intercultural dynamics at work. The following remarks are informed by what teachers in the study abroad program in Berlin articulated about the subject matter, and by my own experience of teaching and working with study abroad students.

\section{Teaching/Learning About the City from a Teacher's/Learner's Perspective}

Teachers' dispositions can be constructed from their personal and professional understanding of study abroad students and the subject of the city, the kind of role for themselves they infer from their understanding of students, and the kind of approach to teaching they derive from their understanding of the city. Various educational philosophies have proposed that the role of the teacher transcends that of the presenters and instructors. Thus, the teaching role should be thought of plurally in the sense that teachers assume specific functions depending on the given dynamic of a teaching/learning situation. Yet, it is not only the situational demands that favor certain roles over others. In light of the students' different cultural backgrounds, teachers 
should adapt their pedagogical considerations to different target groups. Further, the city's manifestations, in particular the meanings and symbolisms they yield, and the interpretation of these by students, may be informed by a different understanding of cities than the teacher's, or, depending on the student's home environment, informed only by vague notions of the city. This dynamic means that in study abroad, effort may be required to facilitate the use of the city as a site of experiential learning. Examples of such efforts are teachers conducting topically focused walking tours or becoming facilitators between study abroad students and cultural protagonists of the city, thereby providing richer and more authentic learning environments.

As for a teacher's approach toward the city, much depends on the teacher's academic training and how the teacher's discipline conceptualizes cities. The variety of academic disciplines yields an array of perspectives on the essence of cities as well as how to employ an intelligible schema to make sense of "the human jungle." Whether a teacher is interested in the form and space of the city, its mores and habits, or the consumption and means of cultural production of its protagonists, the disciplinary focus determines if this teacher will be inspired solely by Weber, Weber and Benjamin or, Benjamin, Lefebvre, Simmel and Weber all together (Parker, 2004). In any case, the choice of approach has an effect on the applicability of experiential teaching. Approaching the city with a focus on its visual cultures, for example, provides more immediate potential for practices of experiential teaching than looking at the city through literature and film, where the engagement between students and the city is overlaid by fictional "texts."

The study abroad student's perspective on the host city can be framed along the coordinates of prior city savvy and knowledge, specific expectations of living and studying in the host city, and engagement with the city's manifold cultural offerings. The host city's novelty and uniqueness stir an excitement which often leads to experiences being much more profound and intriguing, both in terms of leisure and academic engagement. Whether a student notices the lack of public drinking fountains, starts to solely rely on public transportation, or uses bike lanes; whether he or she travels extensively through Europe or observes the new inhabitants of a recently gentrified neighborhood, the city as the site of study abroad provides almost limitless opportunities to engage with it in various ways, to experience it and to cultivate a better understanding of it. Out of the multitude of experiences that stu- 
dents are confronted with by cities and their populations, it is the experiences that do not correlate with prior knowledge that require the student to invest a heightened effort in observing, reflecting, analyzing and synthesizing. It is in this context especially, that aspects of experiential learning can be applied to studying the city's manifestations and meanings on an academic level.

\section{Reflections of a Visual Urban Cultures Teacher}

My remarks will primarily relate to an interview conducted on April 29, 2009 with a professor teaching a course on visual cultures and the urban landscape. Out of the classes offered in the study abroad program at hand, the subject and methodology of this course in particular opened up many opportunities for experiential teaching. The interviewee's comments will disclose further thought on the implications of experiential education in the city. The one-hour interview was conducted in the form of an expert interview, i.e. it was focused on the interviewee's professional reflections on experiential education, and assumed the truth value of his comments (Helfferich, 2009). The interview was conducted in German but the passages cited in this article have been translated into English. The main points raised from the interview analysis will be put into perspective with the responses of other interviewees and then checked against the implications of the models of experiential learning and experiential education.

The interview was structured to illuminate three research questions. First, I was interested in the interviewee's approach to experiential education and the way he understands how his role and his subject might cater to situations of experiential learning. Second, I wanted to tap into his reflections on how to manage the relationship between academic quality and experiential learning, and third, I wanted to learn about best practices for experiential learning and how to address intercultural hurdles that emerged in the course of teaching.

\section{Initiating Experiential Teaching}

The visual cultures course follows an interdisciplinary approach to Berlin as a European city whose physical appearance carries the marks of many historical, and often ideologically antagonistic, political, architectural, artistic, and aesthetic periods. The course attempts to elaborate on the interdependencies between the various modes of human thought and 
activity, and their respective manifestations in the urban landscape. To do so the instructor makes ample use of site visits, city walks, and interviews with artists and local protagonists. While the interviewee did not explicitly refer to his teaching approach as experiential until I used the term, his remarks reveal an intuitive understanding of experiential education and its centrality as a pedagogical method for teaching the city. According to him, successful experiential teaching requires a number of preparations. Students need to understand that the teacher demands their active engagement with the city; the focus of the class supersedes conventional standards of in-class participation. This expectation resonates with that of other interviewed teachers and it concurs with Itin's observation of a general unfamiliarity of students with "being engaged in an educational process that involves their active engagement and participation" (Itin, p.97). Such increased engagement is reflected in the course requirements which call for a final independent project in which students choose one aspect of the city as discussed earlier in the semester for intensive focus. Of all assessed course activities, oral participation carries the greatest weight. Further, to avoid confusion about the demands the students will in the course face, the teacher communicates his expectations more thoroughly as follows:

...of course this is also a learning experience for me as the teacher. In the beginning, the students are very shy, reserved, and cautious. It must be made clear to them that it is not harmful to give wrong answers or to develop ideas which are not close to what will finally be established. One carefully approaches a subject and that creates learning processes. I do emphasize active participation in class which does not mean that I expect a fully-fledged and perfect presentation. I leave that for the final project where I give more time and demand a little more thought and perfection. But in class I want them to be more spontaneous and to take a risk once in a while. Of course, this needs to be prepared for. [...] It is for their own good not in order to please me or to satisfy my duty to grade them.

In this passage, the teacher characterizes the dynamic between himself and his students. He points to the necessity of setting the stage for experiential teaching and learning. This process of preparation and negotiation is a mutual and transactive learning process throughout the course and establishes a com- 
mon ground (Itin, 1999). At the same time this negotiatory process calls for intercultural sensitivity. The interviewee acknowledges the student's reservedness, which is quite typical and prominent at the beginning of a semester. Other respondents similarly acknowledged the presence of this attitude in their classes which, according to them, can be attributed to the cross-cultural encounter that is dramatized by subtle details such as the teacher's accent while speaking English, or more serious misconception such as underestimating the students' ability to critically reflect on their own cultural background.

In addition to emphasizing the importance of oral participation in experiential learning, it is necessary to encourage students to rely on their senses and strategies of meaning making as impulses for experiential and intercultural learning. The threshold to give in to one's subjective observations and interpretations as sources for expanding one's knowledge is high, especially at the outset of one's study abroad sojourn when students are particularly sensitive to aspects of the host culture that are not yet familiar. The search for valid parameters in order to more aptly navigate within the host culture often takes a back seat to the more instant and effortless creation of a comfort zone centered around one's fellow compatriots in the program or their homes, via Internet communication. The interview under discussion, as well as other interviews, revealed a general tendency of program students to avoid failures and risks both in informal and in-class interaction. Preparing, as the interviewee puts it, students to be ready for spontaneity and risk, therefore, requires work on two fronts, both aiming at the establishment of greater certainty.

First, the interviewee repeatedly extends the invitation for active participation in the ongoing class discourse and creates situations that ask for students to observe, characterize, compare and, ultimately, to define the city's images, signs, and symbols. Second, the interviewee recognizes the necessity of providing the students with theoretical knowledge and vocabulary. Only when thus equipped can students observe and interpret with more accuracy and, thereby, do justice to the description of the city while reducing their fear of giving wrong answers.

\section{Reconciling Experiential Learning and Academic Learning}

For a number of reasons the tenets of experiential education and those of academic learning seem to contradict each other. Experiential learning fre- 
quently emerges in progressive education that is associated with adventure and fun. With its stress on the sensory and affective aspects of learning, experiential learning is perceived as forming a somewhat antagonistic relationship with academic learning and certain levels of theoretical reflection. It is not without reason that literature on these two seemingly adverse notions either challenges them, in an attempt to advocate one over the other, or makes a cause for their compatibility (Stavenga de Jong, 2006; Steinberg, 2002).

Theorizing about the city in order to tap into and grasp its complexities is not only necessary to meet academic standards, but also to frame Kolb's so-called cycles of experiential learning. In these cycles students build more informed hypotheses based on reflection on concrete experiences. Asked to explain his use of excursions, the interviewee commented on the strained connection between experiential learning and theoretical reflection:

We have a limited amount of time and we have the city and the city is the star and the object. Of course, a college course needs to be theoretically undergirded, but I try to make this implicit, which means that in the reflection of what one sees, one has to have a theoretical standard. But I think I keep the ball low regarding theoretical discourses and the engagement with different theoretical approaches simply to do justice to the historical and primarily visual object. This is also due to the fact that students come from different academic disciplines. How do they finally benefit from mastering the glass bead game of sign, signifier and signified, if they do not have an overview of the factors that shape a city and that constitute different interests in the city's history? And this is where a different, a historical, maybe even sociological but above all an art-historical moment appears and there is also theory. But this is always, how shall I put it, this theory is always tied to the [visual] objects. I consider these objects as manifests. For example, a graffiti is not only something virtually open to any interpretation. There are indeed conveyed messages. Those can carry a double meaning. They might allow for a certain amount of free interpretation, yet always confined. There is no arbitrariness because everything takes place within certain historical contexts and in certain environments of the city.

This passage presents a way of maneuvering between experiential learning and theoretical reflection. The interviewee calls for the selective appli- 
cation of theory that is dictated by the respective manifestation and/or constitutive force of that manifestation in the city - graffiti, a squatter, or a public square under surveillance. At the same time, such selective application still allows for, if not even enhances, experiential learning as it adds to the development of a more thorough understanding of the city's physical and functional features.

However, this is not to say that teachers and students can jump right into an intensive analysis of the city from session one. The interviewee repeatedly highlights the importance of preparing situations of experiential learning both in terms of sensitizing students to experiential learning techniques and introducing methods of reading the city's open and hidden meanings. Thus, he spends the first half of the semester in the classroom lecturing, providing knowledge, and preparing for the excursions. To put it in his own words:

$[\mathrm{My}]$ approach is to evoke association through images. Not just to look at images, but to create opportunities for comparisons via a multitude of single images which are grouped in blocks. Characterizing, comparing, defining! First of all, you have to provide an input, then complement it with the literature, and then we have the excursions where the students' active role becomes much more prominent. There, you can discuss right away.

The preceding quotes also serve to thwart a common concern that often designates experiential learning as too subjective, too tied to the students' cognitive and emotional responses, and thus, too removed from traditional pedagogical structures to be graded and granted academic credit. Given its subjective nature, experiential learning is sometimes stigmatized as arbitrary. Yet, the interviewee stresses the preparatory phase as a frame for experiential learning within a certain confine, namely, the acquired ability to engage with the city's visual nature and to approach it theoretically.

\section{Experiential Teaching and Learning in Practice}

The following examples of experiential teaching and learning practices have been put to use in courses at the study abroad program under investigation. The goal is to describe their designs and underpinning ideas and to sub- 
sequently reflect on their effect on experiential and intercultural learning.

\section{Walking Through Two City Neigh borhoods}

As mentioned earlier, the interviewee embraces prepared excursions through the city as a way of creating episodes of experiential learning. His excursions take on different formats because they serve different purposes. Yet they share three general characteristics - they follow an interdisciplinary design, try to encourage students to realize the reciprocity between the constitutive forces of history and culture that help to shape the city, and try to deconstruct the notion of urban space as a culturally and socially homogeneous container, in order to acknowledge the internal variety of the city, which is manifested in its neighborhoods (Lefebvre, 1991). For this purpose, nothing is more applicable than having students walk through two neighboring city districts in Berlin. The first has been more recently transformed from a working-class neighborhood into a popular area for tourists and temporarily residing musicians, artists and globetrotters and the second, a traditional immigrant neighborhood. While walking, the students hear languages which are obviously diverse but distinct to the neighborhoods, and they see different but distinct kinds of ethnic food offered on the streets as well as different products displayed on shelves and in window displays that cater to different clienteles. Prior knowledge and/or experience comes into play here. It soon becomes apparent that this is not the transplanted miniature of a Chinatown or a Little Italy as it can be found in America. Though one might conjecture that the students find themselves, then, in a more interspersed model of an immigrant neighborhood, this too is false - at least as it is known from an American contextual perspective. Hypothesis-building starts to set in. Fed by earlier input from teachers about the differences between American and German 19th and 20th century history, and immigrant history in particular, it becomes clear why one can find social housing built in the Bauhaus style of the 1950s and 60s to fill the empty lots left by the bombing of World War II next to Wilhelminian style houses in these sections of the city. One starts to understand how the NeoRomanesque church in the same street can be regarded as a sign indicating an early wave of immigration of Catholics from Silesia. If one juxtaposes the adjacent Turkish Community Center to this church, the diversity of the city's populace is highlighted and poses questions about the coexistence of 
city communities.

And what about the neighborhood with musicians, artists, and globetrotters? How is it possible to transfer, apply, and test the hypotheses developed through the engagement with the immigrant neighborhood? Have the demands of tourists, and more recently arrived residents, already left their imprints on this neighborhood? Once students had the chance to scrutinize the dynamics and specifics of a neighborhood, it was possible to extend their analytical capabilities to other parts of the city and to continue further cycles of experiential learning.

One example of such a continued experiential learning cycle occurred when students tried to frame the interplay of the appearance of a city neighborhood and its dwellers in terms of a set of positive correlations and tried to transplant those to other city neighborhoods. Thus, they attributed the concentration of certain retailers and chain stores to the predominance of immigrants in the neighborhood and, additionally, associated the desolate condition of apartment houses and infrastructure with the overwhelmingly Turkish and Arab neighborhood citizenry. However, the fact that other neighborhoods of Berlin with a less dense immigrant population attract the same retailers and chain stores and that neighborhoods with similar demographics as the aforementioned can have much more intact façades and streets, required students to refine or abandon their hypotheses. They realized that it was necessary to accredit more importance to the formative impact of local actors onto their neighborhood as well as to the different degree of success with which they exert that impact.

\section{Subway Hopping}

One frequent explanation provided by teachers when asked why they do not invest more in the ideas of experiential education is the lack of time. To put it in the words of another interviewee:

Finding the right kind of balance between lecture and discussion and field trips is very difficult, if you only have 30 hours to work with. That's the kind of balance that is a real challenge for me because this course could easily be twice as long as it is now. [...] It's an extra challenge that we have, not just to teach material and to find time to discuss it, but also to get them outside to experience the stuff. So time is really a critical factor here, it's a problem. How do you get all these things in? I could teach the 
course I am doing entirely on the street outside and never give a lecture, or I could do the whole thing in lecture form, or I could teach the whole thing in discussion form. But I need to teach all three, so how do you divide that up? But I definitely believe, and I think it is definitely clear, that if students are coming from Oregon, where they grew up and never left and they finally go abroad and come to a city like Berlin, they should hit the ground running, experience that city, meet its local people ...

The relatively short amount of time available in a study abroad semester seriously hampers one's ability to teach a subject comprehensively. The timeconsuming nature of experiential teaching makes the task even more challenging. Such encounters need to be prepared for, subsequently discussed, and finally evaluated to direct attention to all nodes of the experiential learning cycle. Lecturing, discussing, and field trips must be well planned to safeguard positive learning results. This calls for creativity in both the streamlining of teaching efforts as well as time management to allow for experiential teaching. Collaboration among teachers in the Berlin program has proven time efficient and allowed some field trips that multiple classes might take to be merged, therefore avoiding the visitation of the same site more than once. Additionally, this contributes to an enriched learning environment where differing perspectives contribute to the experience and discussion. Thus, a collaboration between two teachers has often enriched the learning environment by providing two perspectives.

Another creative approach to solving the problem of time shortage is subway hopping. Subway hopping is used for a city excursion designed to remain within the constraints of a four-hour time slot and to focus on different artistic and architectural periods. Without the use of the subway, the excursion might last a whole day. Berlin's public transportation system is elaborate and generally functions quite well. Subways are one of the oldest means of public transportation in Berlin and the architecture of subway stations reflects many different historical and contemporary styles. Experiencing the stations first hand is prepared for in a preceding lecture with PowerPoint presentations. The teacher additionally uses photos and images printed on boards that he presents on the trains in transit between subway stations. In a way the experience is underway even before the actual experience takes place. This is important because the immediate encounter with the subway 
stations lasts only between 30 seconds and a couple of minutes depending on whether students have to hop on the same train with which they arrived or switch and hop onto the next line. During this interval students are asked to solely concentrate on the aesthetic quality of the architecture they perceive. What thoughts and emotions does it evoke? The time period is short. But in it, students are called to heighten their perceptiveness.

In transit again, the students can start articulating and discussing their perceptions before they encounter the next object of comparison in the form of another subway stop. During the follow-up in-class session more time is dedicated to a focused discussion of architectural styles and reflections on the experience. The mode of presentation during the subway rides proves to be worthwhile for students as they gain confidence when it comes to identifying stylistic devices of a certain architectural period. When asked to speculate on why students can better retain content after having been exposed to this experiential teaching method, the interviewee suggested that it might be related to the customary and conditioned ways in which young people engage with video and Internet. The instant and accelerated access to images and information provided by those sources might cater to the fact that the subway hopping does not cause sensory overload but instead is attuned to the students' mode and rate of sensory input. In that sense, the discussed experiential teaching method would not only cater to an exposure to and a reflection on a direct experience, but also to accustomed modes of learning. Likewise, the increased consumption of time often associated with the ability to thoroughly reflect on a subject matter becomes more relative when the process of reflecting on a subject matter is postponed. The various modes of approaching a content - sketchily and condensed as during the subway hopping as well as comprehensive and refined in a classroom discussion or response paper - can enrich the reflection and help it become more nuanced.

\section{The Rap Workshop}

A workshop on rap is part of a course dealing with the impact of pop culture on the modern metropolis. As an event to which the teacher invites a local rapper and producer to talk about his art and to exhibit samples of his music, the workshop incorporates many of the ideas of experiential education. It highlights the transactional element between the rapper and teacher as one unit and the students as the other. The rapper's stories about Berlin's 
rap and hip hop scene reflect how this musical and cultural genre was inspired by American rap artists and how it emulated these role models before it developed into something of its own. The intercultural learning first takes place on a cognitive level in that students come to understand the similarities and differences between American and German rap. Although topics discussed in lyrics might differ, and the English lexicon and syntax lend themselves better to rhymes and neologisms, rap in both countries continues to convey the performer's messages in cleverly rhymed choruses and stanzas.

Subsequently, students are asked to write raps. Although rap is an artistic impression that has its origins in a specific American social and cultural context, today it is part of the American pop culture and experiential repertoire of teenagers and people in their twenties. For pedagogical purposes, dealing with rap is conducive to experiential learning because students already can identify with rap or, at least, relate to the art form. Not only can they fall back on prior knowledge of what rap lyrics are supposed to be like, students also become emotionally invested in learning when they articulate experiences and thoughts in written raps in either English or broken German. The transactional learning between teacher, rapper, and student becomes prominent when students, assisted by the technical equipment of the rapper, perform their raps and reflect on what inspired them. More often than not, this turns out to be something related to the study abroad sojourn, which tends to be the overarching experience already occupying their minds. Often, this leads to broader reflections on Berlin's music scenes and relates those to observations and experiences they have had on field trips to Paris and St. Petersburg.

The rap experience forms new knowledge, provides clues for the teacher on how to refine his experiential teaching and encourages further experimentation with the art form. The invited rapper has repeatedly expressed his admiration for the ease and eloquence with which the American students catch on to his stimuli. This ties in with the teacher's observation that this workshop helps overcome the difficulty of engaging students in a more conscious reflection on their own cultural background. The implicitness of understanding American pop culture obstructs a closer examination, for example, of the Americanization of European cultures. It seems, however, that once reflection is embedded into a familiar context, more distinguished results can be achieved for experiential learning. In this sense, the rap workshop demonstrates how experiential education can mediate the intercultural 
and conceptual differences among the actors involved in its process.

\section{Conclusion}

Cities offer a wide and not yet fully explored range of opportunities for thinking about experiential education and creating situations of experiential learning. The transactional model of experiential education does not invalidate the model of experiential learning. On the contrary, the transactional model enriches our perspective as it draws our attention to the city as a learning object and a learning environment. Also highlighted in the preceding discussion are the intercultural dynamics of its mediation in experiential teaching and learning. The relationships of the actors involved in experiential education and their respective dispositions toward one another and the city, as the object of their negotiations, is complex. Continued conceptualization will be advantageous in further studies. As it has been shown in the interview analysis, implementing the tenets of experiential education theory in practice poses a number of challenges. Students need to be prepared and trained for this mode of learning. It takes effort to reconcile direct experience with more abstract learning. The overall conditions of teaching under time constraints and overloaded program schedules are not always conducive to experiential education.

However, the discussion of the practices in this article provides further thought on how to effectively incorporate components of experiential learning into the teaching of a city. They inspire new ways of handling the challenges involved in experiential learning in the city. These include preparing students for experiential learning by cultivating their analytical and comparative capabilities, undertaking experiential learning within shorter but focused periods of time within alternative learning environments, and managing the level of abstraction within which experiential learning occurs.

The overall response to experiential teaching among those interviewed for this research was positive. Some teachers admitted that they should probably make use of it more often than they do. When asked to elaborate on this hesitation, explanations included the increased effort involved in designing experiential teaching, the interdisciplinary approach that requires further expertise, and the view that experiential learning might be reconcilable with "academic" teaching, but still takes time away from it. Nevertheless, it is my contention that despite certain obstacles, it is still possible to include some aspects of experiential education, for example, by providing for experientially rich learning environments in general, and by reflecting more consciously 
on the intercultural dynamic of the teacher-student relationship. The city's capacity for teaching practices that embrace experiential education has not been exhausted, and calls for more experimentation. It might be possible that the lessons derived from such experimentation will vindicate the use of experience in learning and silence its skeptics.

The usefulness of evaluating the effects of experiential teaching will always remain limited in its significance unless the effectiveness of experiential learning for students is addressed. Hence, further research on students' learning outcomes is needed in order to enhance the effectiveness of experiential teaching and learning together under the roof of experiential education. In the context of teaching and learning about the city, this translates to more theoretically buttressed insights into the effects of the city on students, both as their study abroad environment, and as the object of experiential education.

\section{References}

Brenna, Shannon and Kenneth Cushner (2007). Intercultural Student Teaching - a Bridge to Global Competence. Lanham, MD: Rowman \& Littlefield Education.

Cushner, Kenneth (2004). Beyond Tourism: A Practical Guide to Meaningful Educational Travel. Lanham, MD: Rowman \& Littlefield Education.

Helfferich, Cornelia (2009). Die Qualität qualitativer Daten. 3rd ed., Wiesbaden: Verlag für Sozialwissenschaften.

Itin, Christian M. (1999). Reasserting the Philosophy of Experiential Education as a Vehicle for Change in the 21st Century. The Journal of Experiential Education. 22(2), 91-98.

Kolb, David (1984). Experiential Learning as the Science of Learning and Development. Englewood Cliffs, NJ: Prentice Hall.

Kolb, David, et.al. "Experiential Learning Theory: Previous Research and New Directions." Perspectives on cognitive, learning, and thinking styles. Ed. R.J. Sternberg; J.F. Zhang. NJ: Lawrence Erlbaum, 2000.

Lefebvre, Henri transl. by D. Nicholson-Smith (1991). The Production of Space. Oxford: Blackwell Publishing.

Lutterman-Aguilar, A. and Orval Gingerich. "Experiential Pedagogy for

Study Abroad: Educating for Global Citizenship." Frontiers: The Interdisciplinary Journal of Study Abroad VIII (2002): 207-229.

Parker, Simon (2004). Urban Theory and the Urban Experience. London: 
Frontiers: The Interdisciplinary Journal of Study Abroad

Routledge

Savicki, Victor. "Experiential and Affective Education for International Educators." Developing Intercultural Competence and Transformation. Ed. V. Savicki. Sterling, VA: Stylus Publishing, 2008.

Stavenga de Jong, J.A., et. al. "An exploration of the relationship between academic and experiential learning approaches in vocational education," British Journal of Educational Psychology. 76;1. (2006): 155-169.

Steinberg, Michael “'Involve me and I will understand': Academic Quality in Experiential Programs Abroad." Frontiers: The Interdisciplinary Journal of Study Abroad VIII (2002): 41-82. 